\title{
A Study of the psychological impact of media using modified Beddington- DeAngelis function in the SIR model for contagious diseases
}

\author{
Gurpreet Singh Tuteja
}

Zakir Husain Delhi College, University of Delhi

\begin{abstract}
In this paper, we study mathematically the psychological effect of media on the transmission dynamics of contagious diseases [1]. The SIR model based on compartment theory[18] consisting of three compartments: susceptible, infected and recovered with the transmission function as modified Beddington-DeAngelis function including a parameter governing media awareness is considered. The governing differential equations are defined for the dynamical system. The reproduction number $R_{0}$, of the model, is calculated using the Jacobian matrix method [12] and is found to depend on $\mathrm{m}$ (parameter controlling media) and $\delta$ (a measure of inhibition due to awareness of infected). The stability of the dynamical system at the equilibrium points is discussed. The numerical solution is obtained by varying the introduced parameters of the above-said function and analysed graphically.
\end{abstract}

Keywords: SIR, epidemic, basic reproduction number, media, Beddington-DeAngelis

AMS Classification: 00A71, 34N05

\section{Introduction}

The compartment model theory has been used to understand the transmission dynamics of the contagious diseases like Zeka, HIV, Influenza including Coronavirus COVID19 [19][20][21] using differential equations. Many deterministic models were also developed and applied to understand the dynamics of several infectious diseases [5][6][9][11] [27].

The total population is divided into different compartments based on an individual's stage [34]. The basic SIR model developed by Kermack and Mc Kendrick's introduced three compartments called as $S(t), I(t)$ and $R(t)$, which denote the number of susceptible, contagious (infected and infectious) and recovered/removed individuals at any time $t$, respectively. This model is suitable for diseases which grant permanent immunity after recovery or individual is removed from the system.

The transmission of the disease from the susceptible compartment to the infected compartment is defined using the incidence rate. The bilinear incidence rate $\beta S(t)$ ( $\beta$ is the transmission rate of the disease) has been studied[16][17][18] with the assumption that the susceptible population is large. If the population is saturated with infected individuals, then the number of contacts between infected individuals and susceptible individuals may decrease due to the quarantine of infectious individuals or protective measures taken by susceptible individuals, hence the rate of infection may depend non-linearly to $I(t)[2][3]$. The different kind of saturated incidence function depending on susceptible and infected $\beta S /(1+\alpha S)$ or $\beta S /(1+\alpha I)$ [23], BeddingtonDeAngelis incidence function $\beta S /(1+\alpha S+\beta I)[1][3]$ Crowley-Martin incidence function $\beta S /(1+\alpha S+\beta I+\alpha \beta S I) \quad[7][26][35][36], \quad$ the specific nonlinear incidence function $\beta S /(1+\alpha S+\beta I+\gamma S I)$ $[13][14][15]$ and the non-monotone incidence function $\beta S /\left(1+\alpha I^{2}\right)[35]$ has been studied.

In this paper, we use the SIR model to study the role of media and its psychological/inhibition effect in understanding the dynamics of infectious diseases [10]. The impact of media can't go on increasing forever on the spread of disease and therefore attains saturation. The impact of media on susceptible populations is governed by Holling type II functional response [24][28]. The growth rate of the cumulative density of awareness programs driven by the media is proportional to the number of infective present in the population. Further, awareness about the disease will alert susceptible to isolate them from infective and avoid being infected by forming a separate class [10]. From the cholera epidemic spread in Bari during 1973, [4] introduced a saturated incidence rate $g(I) S$ into epidemic models, where $g(I)$ tends to a saturation level when $I$ is large, $\mathrm{kI} /\left(1+\alpha \mathrm{I}^{2}\right)$ where $k I$ measures the infection force of the disease and $1 /\left(1+\alpha I^{2}\right)$ measures the inhibition effect from the behavioural change of the susceptible individuals when their number increases or from the crowding effect of the infective individuals, also it reduces to bilinear incidence rate when $\alpha=0$. An epidemic model with a specific nonlinear incident rate 
$\mathrm{kI}^{2} \mathrm{~S} /\left(1+\mathrm{I}^{2}\right)$ is studied by[30]. They derived sufficient conditions to ensure that the system has none, one, or two limit cycles. The general incidence rate $\mathrm{kI}^{\mathrm{p}} \mathrm{S} /(1+$ $\left.\alpha \mathrm{I}^{\mathrm{q}}\right)$ was proposed by [25], and studied in [9] [16] [17] [18]. The simple media function $I S /(I+I)$ was studied by [32].

Many other mathematical models have investigated the impact of media coverage on the transmission dynamics of infectious diseases by incorporating a nonlinear function depending on the number of infective individuals $I /(m+I)$ in their transmission [8][31][33]. The function is a continuous bounded function that takes into account disease saturation or psychological effects, it may be noted that in this model the basic reproduction number is independent of $\mathrm{m}$ (media factor).

We propose to study the psychological effect of media, modifying the Beddington-DeAngelis function $\beta S I /(m+\alpha S+\beta I) \quad$ [3], a saturated incidence function considering into account of both susceptible and infected. The denominator has three terms, $m$ denotes the rate with which media is creating awareness causing inhibition or psychological impact.

The paper is organised as follows: In section 2, we state the mathematical equations of the model. The basic reproduction number and stability is discussed at the equilibrium points using Lyapunov function in section 3, disease equilibrium. Section 4, discusses the importance of the parameters related to the psychological impact on susceptible and infected using a numerical solution. Finally, section 5 has concluding remarks.

\section{Formulation}

We consider the SIR model to study the transmission dynamics of contagious disease where the total initial population is divided into three compartments namely, susceptible $S(t)$, contagious $I(t)$ and recovered $R(t)$, where $t$ is the time variable measured in minutes, hours, days, months or years. Let $N$ be the total population in the system at any time $t, S(t)$ denotes the number of susceptible to be infected at any time $t$. The population moves from the compartment of susceptible $S$ to the compartment of infected $I$ depending on the number of contacts infected multiplied by the probability of infection $\beta$ where $\beta I / N$ is the average number of contacts infected with a disease in per unit time $t$. Here, we consider modified BeddingtonDeAngelis to include psychological effect/inhibition due to awareness about susceptible and infected, and the rate with which awareness is being created. The infected $I$ recover with a rate $\gamma$, the recovered means individuals who will return into the class $S$ (susceptible) with a rate $\sigma$, called the rate of recovery. The reciprocal $\sigma^{-1}$ is called the recovery period. $\Lambda$ is called recruitment rate while $\mu$ is the average rate of deaths. $\Lambda$ and $\mu$ describe a model with vital dynamics (endemic model), which has an inflow of births into the class $S$ at a rate $\Lambda$ and outflow (deceased) from any compartment $C$ at a rate $\mu C$. This model is based on the assumptions proposed by [16][19]. The population size is constant and large enough so that the population of each compartment as continuous and all individuals have the same contact rate in the population.

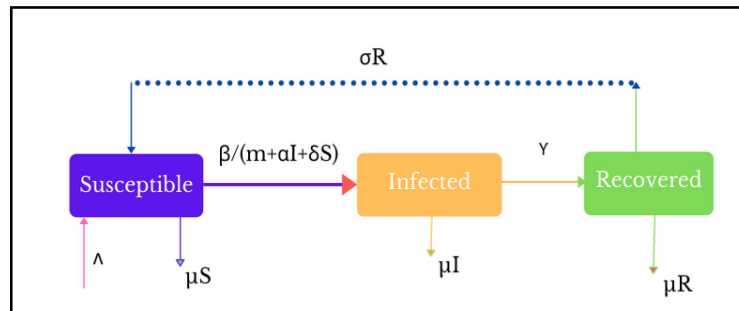

Fig. 1: SIR Model with Vital Dynamics

The governing differential equations of the model are:

$$
\begin{gathered}
\frac{d S}{d t}=\Lambda N-\frac{\beta S(t) I(t) / N}{m+\alpha I(t)+\delta S(t)}-\mu S(t) \\
+\sigma R(t)
\end{gathered}
$$




$$
\begin{aligned}
& \frac{d R}{d t} \\
& =\gamma I(t)-(\mu \\
& +\sigma) R(t)
\end{aligned}
$$

(4)

$$
\text { Where } \quad N(t)=S(t)+I(t)+R(t),
$$

$$
\mathrm{S}(0)=\mathrm{S}_{0} \geq 0, \mathrm{I}(0)=\mathrm{I}_{0} \geq 0, \mathrm{R}(0)=\mathrm{R}_{0} \geq 0 .
$$

The equations (1) - (4) are re-written using dimensionless variables:

$S^{\prime}=S / N, I^{\prime}=I / N, R^{\prime}=R / N$, then omitting dashes, we obtain

$$
\begin{gathered}
\frac{d S}{d t}=\Lambda-\frac{\beta S(t) I(t)}{m+\alpha I(t)+\delta S(t)}-\mu S(t) \\
+\sigma R(t) \\
\frac{d I}{d t}=\frac{\beta S(t) I(t)}{m+\alpha I(t)+\delta S(t)} \\
-(\mu \\
+\gamma) I(t)
\end{gathered}
$$

$$
\begin{aligned}
& \frac{d R}{d t} \\
& =\gamma I(t)-(\mu \\
& +\sigma) R(t)
\end{aligned}
$$

(8)

$$
\text { Where } \quad 1=\mathrm{S}(\mathrm{t})+\mathrm{I}(\mathrm{t})+\mathrm{R}(\mathrm{t}) \text {, }
$$

\section{Disease Equilibrium}

Positivity of the Solution: We show that the model equations (6-8) are biologically and epidemiologically meaningful and well-posed as the solutions of all the stated variables are nonnegative.

Theorem 1: If $S(0)>0, I(0)>0$ and $R(0)>0$, then the solution region $S(t), I(t), R(t)$ of the system of equations $(6-8)$ is always nonnegative.

Proof: Consider each differential equation separately and show that its solution is positive.
Theorem 2: Positivity of recovered: Considering the differential equation (9) of the system

$$
\frac{d R}{d t}=\gamma I(t)-(\mu+\sigma) R(t) \geq-(\sigma+\mu) R(t)
$$

$I(t)$ is positive in time t. On integrating, the solution is $R=R_{0} e^{-\int_{0}^{t}(\sigma+\mu) d t}$. It is clear from the solution that $R(t)$ is positive since $R_{0}>0$ and the exponential function is always positive.

Theorem 3: Positivity of infected population: Considering (6) and that can be rewritten:

$$
\begin{gathered}
\frac{d I}{d t}=\frac{\beta S(t) I(t)}{m+\alpha I(t)+\delta S(t)}-(\mu+\gamma) I(t) \\
\geq-(\mu+\gamma) I(t) .
\end{gathered}
$$

On, integrating the solution is $I=I_{0} e^{-\int_{0}^{t}(\mu+\gamma) d t}$. It is clear from the solution that $\mathrm{I}(\mathrm{t})$ is positive since $I_{0}>0$ and the exponential function is always positive.

Theorem 4: Positivity of susceptible population: Finally, we consider the differential equation (5):

$$
\begin{aligned}
& \frac{d S}{d t}=\Lambda-\frac{\beta S(t) I(t)}{m+\alpha I(t)+\delta S(t)}-\mu S(t) \\
& +\sigma R(t) \\
& \geq-\frac{\beta S(t) I(t)}{m+\alpha I(t)+\delta S(t)} \\
& -\mu S(t)
\end{aligned}
$$

$\Lambda$ is the rate of birth and $R(t)$, being positive, we can write as:

$$
\frac{d S}{S(t)}=-\left(\frac{\beta I(t)}{m+\alpha I(t)+\delta S(t)}+\mu\right) d t
$$

On, integrating the solution is $S=$ $S_{0} e^{-\int_{0}^{t}\left(\frac{\beta I(t)}{m+\alpha I(t)+\delta S(t)}+\mu\right) d t}$. It is clear from the solution that $S(t)$ is positive since $S_{0}>0$ and the exponential function is always positive.

The model equations (5-8) are biologically and epidemiologically meaningful and well-posed as 
the solutions of all the state variables are bounded. As

$$
\frac{d S}{d t}+\frac{d I}{d t}+\frac{d R}{d t}=0
$$

On adding Eqn. (5)-(7), we obtain

$$
\Lambda-\mu S(t)=0
$$

Therefore, the feasible region for the system is given by $\left(\mathrm{S}^{*}, \mathrm{I}^{*}, \mathrm{R}^{*}\right)$

$$
\begin{gathered}
\mathrm{S}^{*}=\frac{\Lambda}{\mu}, \mathrm{I}^{*}=0, \mathrm{R}^{*}=0, \\
\omega=\left[\left(S^{*}, I^{*}, R^{*}\right) \in R^{3+}: S^{*}+L^{*}+R^{*} \leq \frac{\Lambda}{\mu}\right.
\end{gathered}
$$

Therefore, $\left(\frac{\Lambda}{\mu}, 0,0\right)$ is the Disease Free Equilibrium (DFE) point. Hence, it sufficient to consider solutions in the region $\omega$. The solutions of the initial value problem starting in $\omega$ and defined by (5) - (7) exist and are unique on a maximal interval. Since the solution remains bounded in the positively invariant region $\omega$, the maximal interval defined is $[0,1)$. So, the initial value problem is both well-posed and is positive. The above system always has a disease-free equilibrium:

$$
\left(\frac{\Lambda}{\mu}, 0,0\right)
$$

The reproduction number $\mathrm{R}_{0}$ can be computed using the Jacobian Matrix method. The characteristic polynomial is:

$-\frac{(\lambda+\mu)(-\beta \Lambda+(\gamma+\lambda+\mu)(\alpha \Lambda+m \mu))(\lambda+\mu+\sigma)}{\alpha \Lambda+m \mu}$

The Eigenvalues are: $\quad \lambda_{1}=-\mu$,

$$
\begin{gathered}
\lambda_{2}=\frac{\beta \Lambda-\alpha \gamma \Lambda-m \gamma \mu-\alpha \Lambda \mu-m \mu^{2}}{\alpha \Lambda+m \mu} \\
\lambda_{3}=-\mu-\sigma .
\end{gathered}
$$

As all the Eigen-values are negative for a system to be asymptotically stable, so

$$
\frac{\beta \Lambda-\alpha \gamma \Lambda-m \gamma \mu-\alpha \Lambda \mu-m \mu^{2}}{\alpha \Lambda+m \mu}<0,
$$

We obtain

$$
R_{0}=\frac{\beta \Lambda}{(\alpha \Lambda+m \mu)(\gamma+\mu)}<1
$$

The basic reproduction number $\mathrm{R}_{0}$ depends on both $\mathrm{m}$ and $\alpha$ (coefficient of $I(t)$ ) and is independent of $\delta$, coefficient of $S(t)$. Thus, the awareness about the infected has a psychological impact on the growth of the disease and can be meticulously used to curb its spread (function being saturated).

Consider the Lyapunov function [2]:

$$
V=W_{1}\left(S-S^{*} \log \frac{S}{S^{*}}\right)+W_{2}\left(I-I^{*} \log \frac{I}{I^{*}}\right)
$$

Substituting the value of $\dot{S}$, and $\dot{I}$ from equation (5) and (6)

$$
\begin{aligned}
\dot{V}=W_{1}\left(S-S^{*}\right. & \left(\frac{\Lambda}{S}-\frac{\beta I}{m+\alpha I+\delta S}-\mu\right) \\
& +W_{2}\left(I-I^{*}\right)\left(\frac{\beta I}{m+\alpha I+\delta S}-(\mu\right. \\
& +\gamma))
\end{aligned}
$$

Let the equilibrium points be

$$
\begin{aligned}
& \mu=\frac{\Lambda}{S^{*}}-\frac{\beta I^{*}}{m+\alpha I^{*}+\delta S^{*}}, \mu+\gamma \\
& =\frac{\beta I^{*}}{m+\alpha I^{*}+\delta S^{*}} \\
& =\frac{W_{1} m \beta\left(I-I^{*}\right)\left(S-S^{*}\right)-W_{1} \beta^{2}\left(S^{*} I-S I^{*}\right)\left(S-S^{*}\right.}{(m+\alpha I+\delta S)\left(m+\alpha I^{*}+\delta S^{*}\right)} \\
& -W_{1} \frac{\Lambda\left(S-S^{*}\right)^{2}}{S S^{*}} \\
& +\frac{W_{2} m \beta\left(I-I^{*}\right)\left(S-S^{*}\right)+W_{2} m \alpha \beta\left(S^{*} I-S I^{*}\right)\left(I-I^{*}\right)}{(m+\alpha I+\delta S)\left(m+\alpha I^{*}+\delta S^{*}\right)} \\
& \dot{V} \leq 0 \text { for } S<S^{*}, I<I^{*}, S I^{*}<S^{*} I, W_{1}=1, W_{2}=-1 \\
& \text { and also for } S=S^{*}, I=I^{*}, \dot{V}=0 \\
& \text { Therefore, by La Salle's Invariance principle [12], } \\
& \text { the endemic system is globally asymptotically } \\
& \text { stable [22][29]. }
\end{aligned}
$$




\section{Numerical Solution}

We solve dimensionless differential equations (5) to (7) numerically using the NDSolve function of Wolfram Mathematica. The numerical solutions of $S(t), I(t)$ and $R(t)$, are plotted for different values of parameters viz. $\mathrm{m}, \alpha$ and $\delta$. A typical view of the solutions of $S(t), I(t)$ and $R(t)$ for the parameters: $t=50, \Lambda=\mu=.01, \gamma=0.13, \sigma=.011, \delta=1$, $\alpha=1$ and $m=.01, .1,1$ and 5 (Fig. 2-5). As $m$ (awareness through media about infected) increases, the peak of the infected $I(t)$ is reduced giving a positive relation between $\mathrm{m}$ and $I(t)$. The psychological effect of the media $m$ plays an important role in controlling the spread of the disease
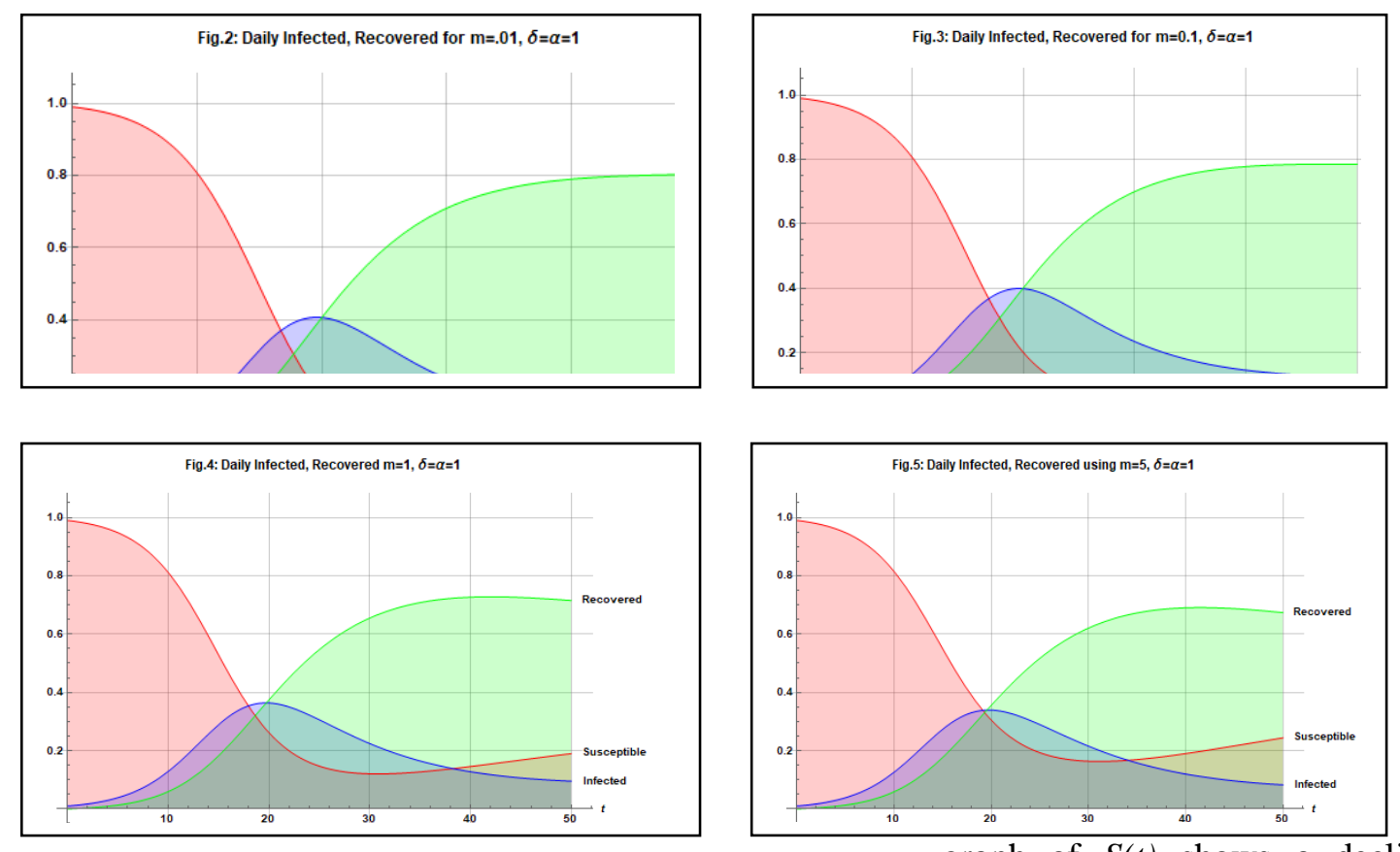

graph of $S(t)$ shows a decline in the numbers of susceptible due to inhibitive or psychological measures

The effect of $\alpha$ (coefficient of $I(t)$ ), is varied from .01 to 5. The values of $\alpha=.01,0.1,1$ and 5, as it increases, the graph of infected $I(t)$ also increases (Fig. 6-9). The by the susceptible due to the awareness created about infected and hence the recovery is also improved (graph of $R(t)$ attains a new peak).
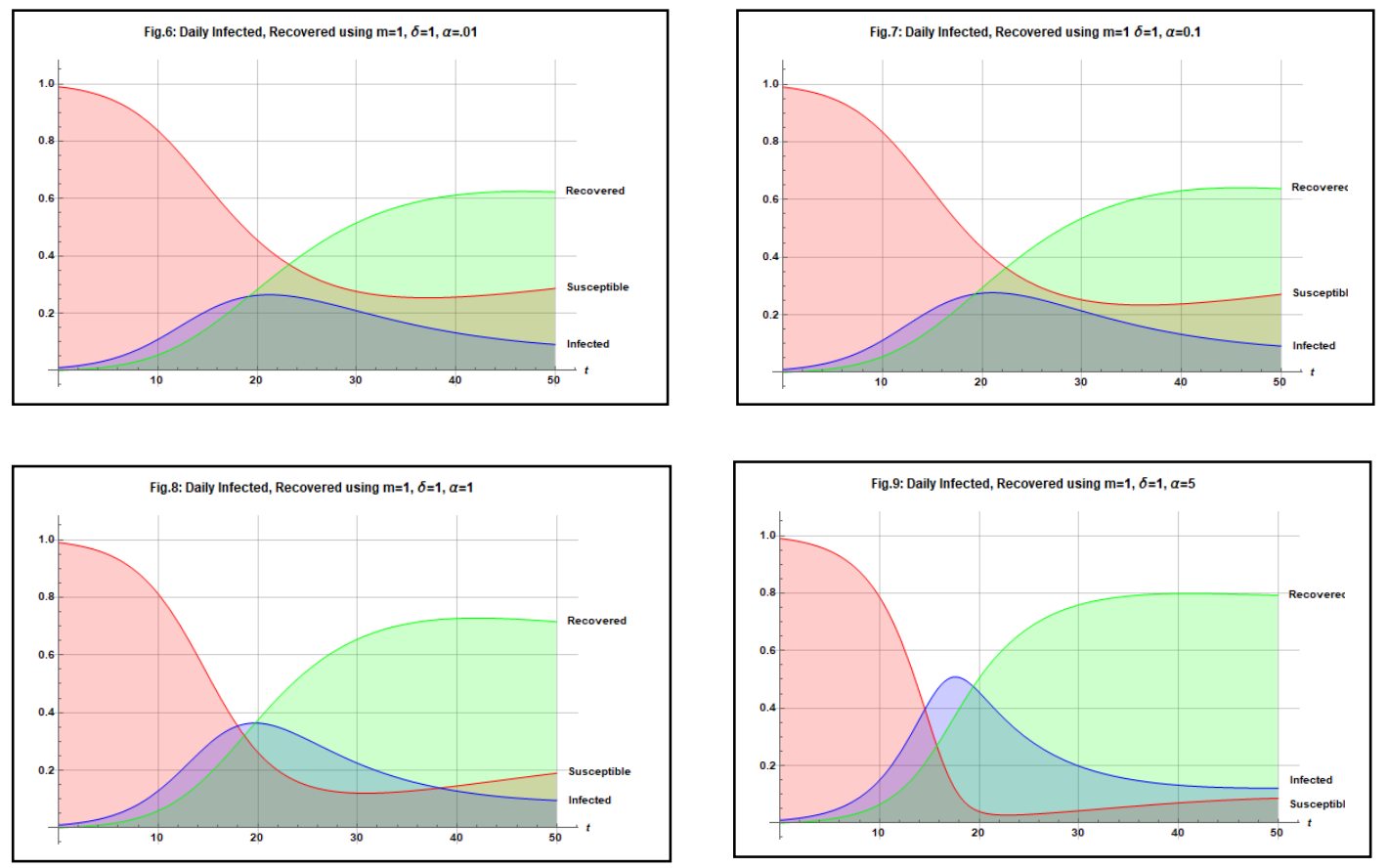
The effect of $\delta$ (Coefficient of $S(t)$ ) is studied by plotting graph of $S(t), I(t)$ and $R(t)$ for different values of $\delta=.01,0.1,1$ and 5 (Fig. 10-13). It has been found that as $\delta$ increases, a measure of inhibitory or psychological measures by creating awareness about susceptible, the graph of susceptible drops quickly with time while recovery is also improved.
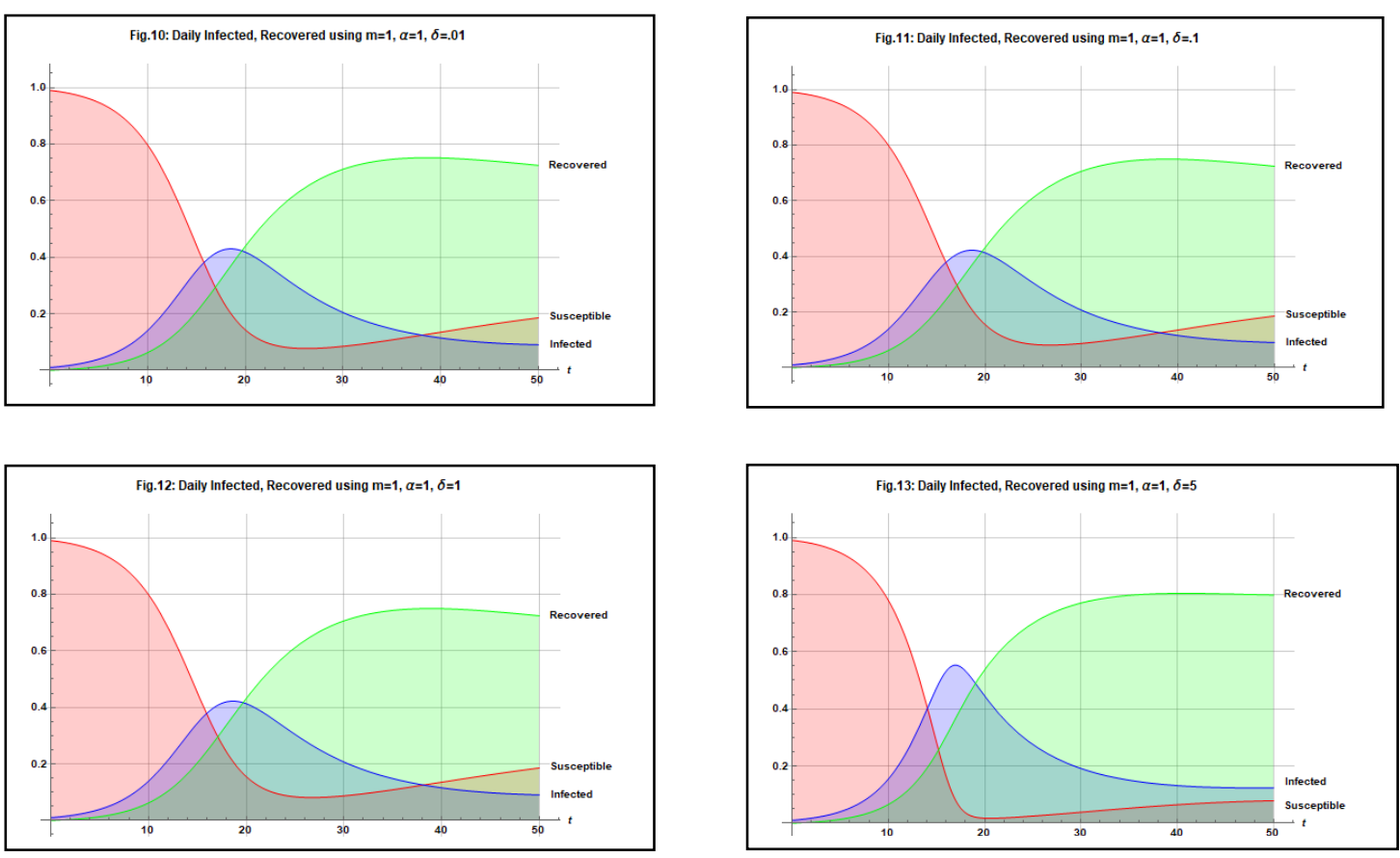

Bibliography

\section{Conclusion}

The model in this study is based on compartment theory, as a SIR model with the transmission of disease based on Beddington-DeAngelis incidence function. The modified Beddington-DeAngelis incidence function is studied for understanding the psychological effect of media and consequently psychological effect or inhibitory measures taken by susceptible and infected to control the contagious diseases. It has been found that the media plays a significant role in creating awareness or psychological effect in the dynamics of contagious disease in a population. The inhibitory effects of creating awareness about infected create psychological effects which can control the growth of the disease to a limit as the function itself is saturated. The basic reproduction number $R_{0}$ is calculated using the characteristic equation and found to depend on the media and the factor $\alpha$ which is the inhibitive psychological measure by creating awareness about infected by media. The equilibrium points for the dynamical system are computed and found to be asymptotically stable using the Lyapunov method.
[1] Beddington, J R. "Mutual interference between parasites or predators and its effect on searching efficiency." J. Anim. Ecol. 44 (1975): 331-341.

[2] Bentaleb, Dounia and Amine, Saida. "Lyapunov function and global stability for a two-strain SEIR model with bilinear and non-monotone incidence." International Journal of Biomathematics 12.02 (2019): 1950021.

[3] Cantrell, R S and Cosner, C. "On the dynamics of predator-prey models with the Beddington-DeAngelis functional response." J. Math. Anal. Appl 257 (2001): 206-222.

[4] Capasso, Vincenzo and Gabriella Serio. "A generalization of the Kermack-Mckendrick deterministic epidemic model." Mathematical Biosciences 42.1-2 (1978): 43-61.

[5] Cheng, Y., Wang, J. and Yang, X. "On the global stability of a generalized cholera epidemiological model." J. Bio. Dyn 6.2 (2012): 1088-1094. 
[6] Chitnis, N., Cushing, J.M. and Hyman, J.M. "Bifurcation analysis of a mathematical model for malaria transmission." SIAM Journal of Applied Mathematics 67.24-45 (2006).

[7] Crowley, P H, Martin, E K. "Functional responses and interference within and between year classes of a dragonfly population." J. North. Am. Benth. Soc 8 (1989): 211-221.

[8] Cui, J, Sun, Y, Zhu, H: " The impact of media on the control of infectious diseases." Journal of Dynamics and Differential Equations 20 (2008): 31-53.

[9] Derrick, W R and Driessche, P Van Den. "A disease transmission model in a non-constant population." Journal of Mathematical Biology 31.5 (1993): 495-512.

[10] Dubey Balram, Dubey Preeti, Dubey Uma S. "Role of media and treatment on a SIR model." Nonlinear Anal. Model. Control 21.2 (2015): 185-200.

[11] Egbetade, S.A. and Ibrahim, M.O. "Modeling the impact of BCG vaccines on tuberculosis epidemics." Mathematical Modelling and Applications 9.1 (2014): 49-55.

[12] Hale, J.K. Ordinary Differential Equations. Second. Florida: Kriege Publishing Company, 1980.

[13] Hattaf K, Mahrouf M, Adnani J, Yousfi N. " Qualitative analysis of a stochastic epidemic model with a specific functional response and temporary immunity Qualitative analysis of a stochastic epidemic model with a specific functional response and temporary immunity." Physica A 490 (2018): 591-600.

[14] Hattaf K, Yousfi N. "Global dynamics of a delay reaction-diffusion model for viral infection with a specific functional response." Comput. Appl. Math. 34 (2015): 807-818.

[15] Hattaf K, Yousfi N, Tridane A. "Stability analysis of a virus dynamics model with general incidence rate and two delays." Appl. Math. Comput. 221 (2013): 514-521.

[16] Hethcote H.W., Levin S.A. "Periodicity in epidemiological models." Gross L., Hallam
T.G., Levin S.A. Applied Mathematical Ecology. Berlin: Springer-Verlag, 1989. 193.

[17] Hethcote Herbert W, Driessche P Van Den. "Some epidemiological models with nonlinear incidence." Journal of Mathematical Biology 29.3 (1997): 271-287.

[18] Hethcote, H. "The Mathematics of Infectious Diseases." SIAM Review 42.4 (2000): 599653.

[19] Kermack, W. O. and Mckendrick, A. G. "Contribution to the Mathematical Theory of Epidemics (Part 1)." Proc. R. Soc. Lond. B. Biol. Sci. 138 (1932): 55-83.

[20] Kermack, W. O. and Mckendrick, A. G. "Contribution to the Mathematical Theory of Epidemics (Part II)." Proc. R. Soc. Lond. B. Biol. Sci. 141 (1932): 94-112.

[21] Kermack, W. O., McKendrick, A. G. "A contribution to the mathematical theory of epidemics. "Proceedings of the Royal Society of London A: Mathematical, physical and engineering science 115 (1927): 700-721.

[22] Li M Y, Muldowney J S. "Global stability for the SEIR model in epidemiology." Math. Bioscience 125 (1995): 155-164.

[23] Liu X, Yang L. "Stability analysis of an SEIQV epidemic model with saturated incidence rate." Nonlinear Analysis: Real World Applications 13.6 (2012): 2671-2679.

[24] Liu, Rongsong \& Wu, Jianhong \& Zhu, Huaiping. (2007) "Media/Psychological Impact on Multiple Outbreaks of Emerging Infectious Diseases." Computational and Mathematical Methods in Medicine 8.3 (2007).

[25] Liu, Wei-min, Levin, Simon A and Iwasa, Yoh. "Influence of nonlinear incidence rates upon the behavior of sirs epidemiological models." Journal of Mathematical Biology 23.2 (1986): 187-204.

[26] Liu, X Q, Zhong S M, Tian B D, Zheng F X. "Asymptotic properties of a stochastic predator-prey model with Crowley-Martin functional response." J. Appl. Math. Comput. 43.1-2 (2013): 479-490.

[27] Madubueze, Chinwendu \& Kimbir, Anande \& Aboiyar, Terhemen. "Global Stability of Ebola Virus Disease Model with Contact 
Tracing and Quarantine. ." Transactions of The Nigerian Association of Mathematical Physics 3 (2018): 75-82.

[28] Mishra A. K., Sharma Anupma and Singh Vishal. "Effect of awareness programs in controlling the prevalence." J. Biol. Syst. 19.2 (2011): 389-402.

[29] Mishra, Bimal Kumar and Jha Navnit. "SEIQRS model for the transmission of malicious objects in the computer network." Applied Mathematical Modelling 34.3 (2010): 710-715.

[30] Ruan, Shigui and Wang, Wendi. "Dynamical behavior of an epidemic model with a nonlinear incidence rate." Journal of Differential Equations 188.1 (2003): 135163.

[31] Rudnicki, R. "Long-time behaviour of a stochastic prey-predator modeL." Stochastic Processes and their Applications 108 (2003): 93-107.

[32] Sun C, Yang W, Arino J, Khan K. "Effect of media-induced social distancing on disease transmission in a two patch setting." Mathematical Biosciences 230.2 (2011): 87 95.

[33] Tchuenche, JM, Dube, N, Bhunu, CP, Smith, RJ, Bauch, CT. "The impact of media coverage on the transmission." BMC Public Health 11.S5 (2011).

[34] Wang J J, Zhang J Z, Jin Z. "Analysis of an SIR model with bilinear incidence rate." Nonlinear Anal. Real World Appl 11.4 (2010): 2390-2402.

[35] Xiao, Dongmei and Ruan, Shigui. "Global analysis of an epidemic model with nonmonotone incidence rate.". Mathematical biosciences 208.2 (2007): 419-429.

[36] Zhou, X and Cui, J. "Global stability of the viral dynamics with Crowley-Martin functional response." Bull. Korean Math. Soc. 48.3 (2011): 555-574. 